\title{
High Resolution Imaging of White Matter Structures in Stroke
}

Rishma Vidyasagar ${ }^{1}$, Robert Smith ${ }^{2}$, Fernando Calamante ${ }^{2}$, Gemma Lamp $^{1,3}$, Peter Goodin ${ }^{1}$, Leeanne Carey Lea $^{1,3}$

${ }^{1}$ Florey Institute of Neuroscience and Mental Health, Neurorehabilitation and Recovery, Melbourne, Australia,

2 Florey Institute of Neuroscience and Mental Health, Advanced Imaging, Melbourne, Australia \section{UNIVERSITY}

${ }^{3}$ La Trobe University, School of Allied Health, Melbourne, Australia

\section{Background and Aim:}

$>$ Diffusion magnetic resonance imaging (MRI) is used routinely in clinical imaging to visualise the location and extent of tissue damage due to a stroke and to visualise white matter structures in the brain.

$>$ Applications of diffusion imaging in stroke recovery has been limited and slow to advance.

$>$ This is partly due to difficulty in reconstructing tracts with a high spatial resolution to visualise subtle changes in white matter structures. It has been shown that advancing diffusion image processing from the standard diffusion tensor model to convoluted spherical deconvolution method is more accurate in stroke recovery ${ }^{1}$.

The aim of this work is to apply novel advanced diffusion image processing to visualise white matter structures with high resolution in the brain of stroke patients. This novel contrast, known as track density imaging (TDI)/22, subsamples tracts from the original acquisition resolution.

\section{Method:}

- Data from one healthy and 2 randomly chosen patients that were a subset of a larger longitudinal stroke study ${ }^{3}$ are shown. Both patients were $>3$ months post stroke, and had lesions either in cortical or subcortical sensory regions.

- Diffusion data was acquired on a 3T Siemens Trio scanner ( $b=3000,60$ directions, $2.5 \mathrm{~mm}$ isotropic resolution).

- MRTrix3 (www.mrtrix.org) software was used to process diffusion data. TDI tracts were created at a $1 \mathrm{~mm}$ isotropic resolution. Directionally encoded colour maps of fractional anisotropy (FA-DEC) and TDI (TDI-DEC) were created.

Results:

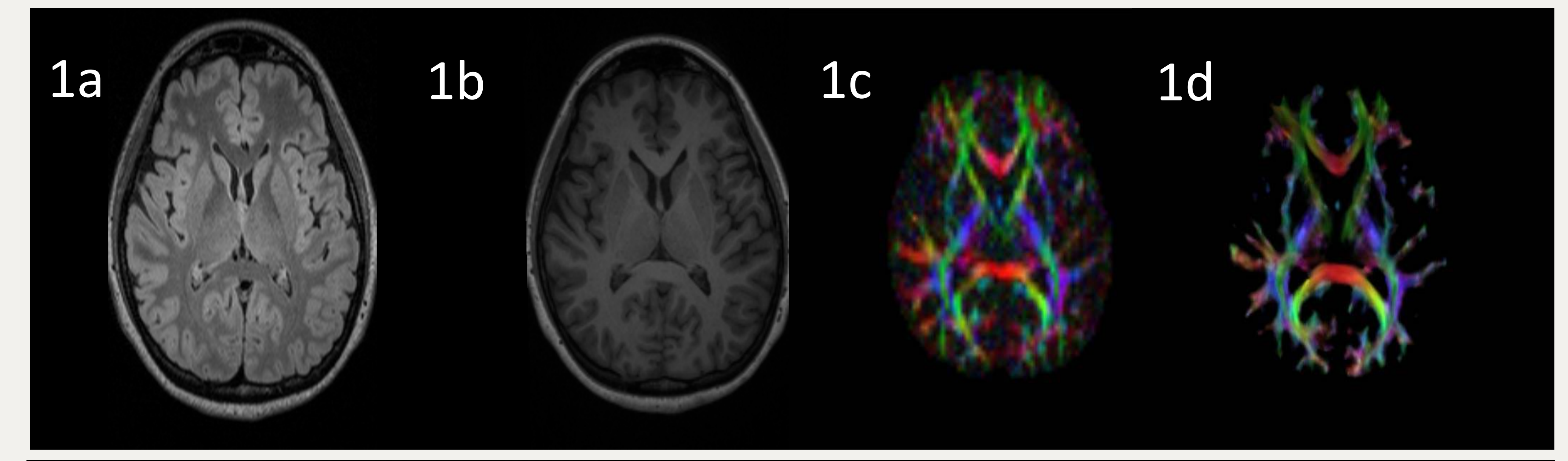

Figure 1: Example from a healthy subject ( $F, 41$ years). FLAIR (1a) and T1 (1b) images showing no lesions. FA -DEC (1c) compared to the TDI-DEC (1d).

Figure 2: Example from a patient $(F, 59$ years) with a subcortical lesion and somatosensory deficit as seen in the FLAIR

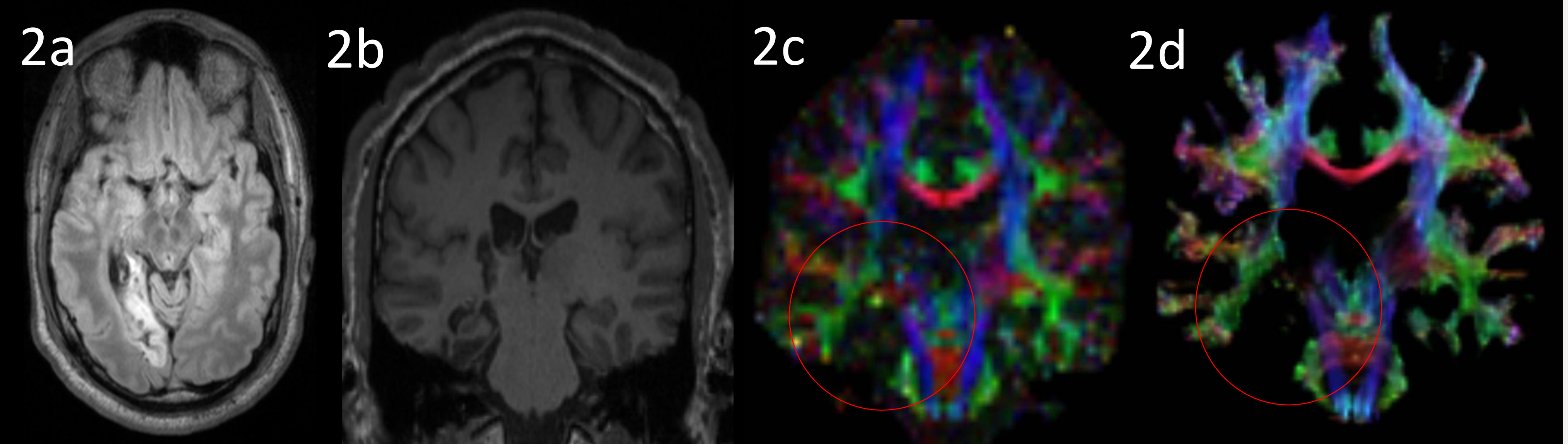
(2a). FA -DEC (2c) maps don't clearly reflect the lesioned affected white matter structures (red circles) compared to the TDI-DEC. These affected structures are important projections that lead to the somatosensory areas.

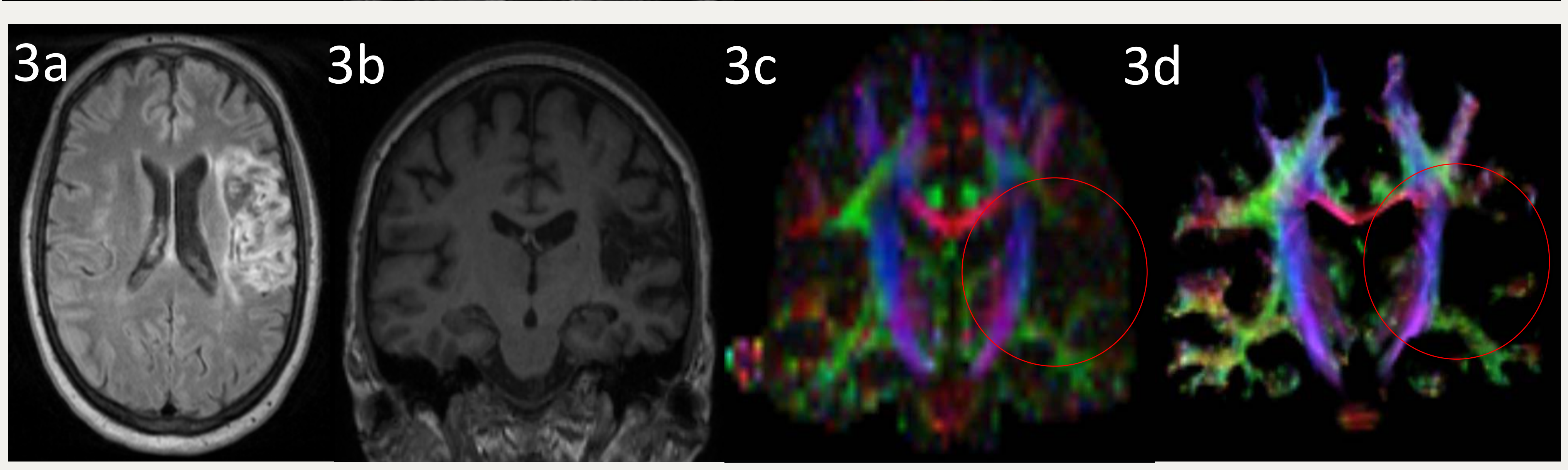

Figure 3: Example from a patient with a cortical lesion (3a) and somatosensory deficits. FA-DEC (3c) maps do not highlight the affected white matter tracts (red circles) as effectively as the TDI - DEC maps. A thinning of the superior longitudinal fasciculus can be observed in $3 \mathrm{~d}$ which is linked to sensorimotor processing.

\section{Conclusions:}

TDI-DEC provide a clearer, high resolution visualisation of the impact of a stroke lesion on white matter tracts. In the examples shown, patients with somatosensory deficits had effects on sensorimotor related tracts that were visible from the TDI maps. 